\title{
FUZZY SET AND PATTERN RECOGNITION AND DATA ANALYSIS
}

\section{Hans-Jürgen Zimmermann, RWTH Aachen}

Fuzzy technology has become widely accepted as a tool to solve problems for which traditional methods are not quite applicable. In the past most of the applications that became known, were in the area of control engineering. These were knowledge-based approaches to control problems. In the recent past an area has emerged which seems to be of even higher importance than fuzzy control, that of fuzzy data analysis. By contrast to fuzzy control in this area algorithmic fuzzy approaches are being used as well as knowledge-based approaches. The main goal is to reduce complexity and to recognize structures.

This reduction of complexity achieves in a number of steps:

1. In modelling the problem or describing the process the first abstraction takes place.

2. In feature analysis the number of features used to describe objects is further reduced.

3. On the basis of these features classes (classifiers) are designed, which eventually reduce the complexity to a degree acceptable for human perception.

4. These classes are then used in the area of classification to structure observed masses of data.

Traditionally methods such as structured modelling, discriminant - and factor - analysis, cluster analysis etc. are used to achieve the goals. 